\title{
Formalising and Verifying Reference Attribute Grammars in Coq
}

\author{
Max Schäfer, Torbjörn Ekman, and Oege de Moor \\ Programming Tools Group, University of Oxford, UK \\ \{max.schaefer, torbjorn.ekman, oege.de.moor\}acomlab.ox.ac.uk
}

\begin{abstract}
Reference attribute grammars are a powerful formalism for concisely specifying and implementing static analyses. While they have proven their merit in practical applications, no attempt has so far been made to rigorously verify correctness properties of the resulting systems. We present a general method for formalising reference attribute grammars in the theorem prover Coq. The formalisation is supported by tools for generating standard definitions from an abstract description and custom proof tactics to help automate verification. As a small but typical application, we show how closure analysis for the untyped lambda calculus can easily be implemented and proved correct with respect to an operational semantics. To evaluate the feasibility of our approach on larger systems, we implement name lookup for a naming core calculus of Java and give a formal correctness proof of the centrepiece of a rename refactoring for this language.
\end{abstract}

\section{Introduction}

Verifying program analyses and transformations is hard, even with the assistance of modern proof tools. Previous work [17|18] has followed standard practice in the compilers community by focussing on simple intermediate languages, where issues like complex scoping have been transformed away. For verifying optimisations in a batch compiler this approach is successful and adequate.

However, many modern development tools such as refactoring editors and code checkers operate at source level, and not on an intermediate language. This requires the implementation of analyses and transformations that align with the 'middle' phases of a compiler that deal with name binding, type checking and complex control flow structures, which are often the most intricate and error prone [106].

This paper presents a framework for verifying analyses and transformations on source, consisting of a specification formalism for source-level analyses and transformations, namely Circular Reference Attribute Grammars, and an embedding of that formalism in Coq. Our embedding is supported by tools that reduce the tedium of working with a complex language definition and allow proofs to be conducted at a high level of abstraction.

The particular variant of attribute grammars we have chosen is the basis of the JastAdd system [7]. It enhances standard attribute grammars [16] with a notion of node identity (reference attributes), and the ability to express least fixed point computations (circular attributes). Both these extensions are well-known: most industrial attribute

G. Castagna (Ed.): ESOP 2009, LNCS 5502, pp. 143-159. 2009.

(C) Springer-Verlag Berlin Heidelberg 2009 
grammar systems support reference attributes [123], and the idea of circular attributes goes at least back to Farrow [8].

The success of JastAdd [28] amply demonstrates the power of circular reference attribute grammars and their practical usability. Particularly relevant are its use in the implementation of JastAddJ, a full, highly compliant Java compiler [7], and in the construction of a refactoring framework for Java [24].

While it is well-known that pure attribute grammars can be regarded as non-strict functional programs [15], reference attributes and circular attributes require a more sophisticated translation. We demonstrate that these complications can be handled gracefully by generating boilerplate definitions and judicious use of proof strategies.

The result is a completely general framework for conducting proofs of analyses and transformations that deal with the full complexity of modern source languages. We illustrate its use on two examples: verification of a closure analysis, and the core of the refactoring framework of [24]. The complete formalisation including mechanised proofs is available for download [25]. Major contributions of this paper are:

- The first generic framework for mechanised proofs of the correctness of program analyses and transformations specified at source level.

- Tool support for conducting such proofs in Coq, at a high level of abstraction.

- A substantial case study that illustrates how our framework copes with the complex scoping issues in modern object-oriented languages, an issue that has hitherto not been considered in a formal setting.

- Evidence that proofs in our framework are scalable: non-trivial extensions to the object language require only modest additions to the proofs themselves.

The paper is structured as follows. First we introduce the notion of circular reference attribute grammars in Sec.2. Next we show in Sec. 3 how this variant of attribute grammars is formalised in Coq. Sections 2 and 3 use a simple closure analysis as a running example. As a more substantial case study, we describe a formalisation of name analysis in an object-oriented language in Sec. 4 and show how it can be used to give a correctness proof of the central component of the Rename refactoring. Section 5 evaluates the development effort for both examples, and investigates scalability issues. Related work is discussed in Sec. 6, and we conclude in Sec.7.

\section{Reference Attribute Grammars}

Attribute grammars [16] are a high-level formalism for declaratively specifying and implementing programs working on trees, in particular programming language processors such as compilers. Nodes of the underlying trees are endowed with attributes defined in terms of other attributes on the same node, or attributes on the surrounding nodes.

As originally proposed, attribute grammars make no special provisions for non-local dependencies, i.e. attributes that are defined in terms of other attributes on nodes far away in the tree. This situation commonly occurs in the static analysis of programming languages: For example, to typecheck the use of a variable, information about its type is needed, which has to be obtained from its declaration arbitrarily far away in the tree. 
Solutions such as the explicit construction of symbol tables tend to become quite complicated, compromising the conceptual clarity of the underlying approach. Many extensions have been proposed to remedy this and similar problems, a particularly simple one being Reference Attribute Grammars, or RAGs [11], which are closely related to Remote Attribute Grammars [3]. The idea here is to allow attributes to evaluate to node references, on which other attributes can be evaluated in turn, thus superimposing a graph structure on the abstract syntax tree. This alleviates the need for secondary structures such as symbol tables and enables the use of the abstract syntax tree as the only data structure. RAGs become especially useful when combined with circular attributes, i.e. attributes defined by recursive equations; the combined formalism of Circular Reference Attribute Grammars, or CRAGs [20], will be our focus in this paper.

To introduce the key features of attribute grammars in general and reference attribute grammars in particular, we show how to implement closure analysis for the untyped lambda calculus. Closure analysis is a static analysis that predicts the reduction behaviour of terms. Take, for example, the term $(\lambda f .(f I)(f K))(\lambda x . x)$ where $I=\lambda a . a$ and $K=\lambda b . \lambda c . b$. Closure analysis will be able to predict that during reduction $x$ can only be bound to the terms $I$ and $K$; we say that the set of closures of (the single occurence of) $x$ is $\{I, K\}$. For a discussion of the uses of closure analysis we refer to the literature [26|22], we concentrate on how to compute the set of closures using an attribute grammar.

\subsection{Abstract Syntax Trees}

Our object language is the simply typed lambda calculus with its reduction relation $t \Downarrow v$, indicating that term $t$ evaluates to value $v$. The definitions of syntax and reduction relation are standard and we elide them for brevity.

Lambda terms are represented as abstract syntax trees (ASTs): Every node in an AST corresponds to a nonterminal of the grammar (here always term). Its children may either be terminals or other nodes, corresponding to a particular production in the grammar. In our example, a term node is either a variable node with a single identifier as its only child; or an abstraction node with an identifier (the bound variable) and a term node as its children; or an application node with two term nodes as its children. A node represents a subterm at a certain position, hence in the example the two occurrences of variable $f$ will be represented by different nodes, albeit with the same content (namely the term $f$ ).

We want to implement an attribute closures that, for each node in the abstract syntax tree of a lambda term, returns the set of possible closures it could evaluate to. We want to prove a coherence result to show that our closure analysis agrees with the reduction relation and correctly predicts reduction outcomes:

Lemma 1 (Coherence). For any lambda term $t$ and closure $v$, if $t \Downarrow v$ then there is some node $n d \in$ closures $(t)$ such that $v$ is the content of $n d$.

\subsection{The Attributes}

To start with, we need to define a parameterised lookup attribute which looks up a name from a position within the term. Proceeding outwards, it returns a reference to 
the innermost enclosing lambda abstraction binding that name, if there is any. Thus, the result of looking up a name $y$ at a node nd depends on the context of the node:

- if nd is the body of a lambda abstraction binding a variable $\mathrm{x}$, we compare $\mathrm{y}$ and $\mathrm{x}$; if they are the same, nd's parent node (the lambda abstraction) is returned as result; otherwise, we proceed with the lookup of $y$ from the parent node

- if nd is the left child of an application node, lookup proceeds outwards

- if nd is the right child of an application node, lookup proceeds outwards

These three cases correspond to the clauses of the definition of name lookup as an inherited attribute, which is given in Fig. 1. using pseudocode. In these equations, this refers to the node lookup is invoked on, whereas parent is that node's parent node. The equations are defined by pattern matching on the position of this relative to parent, which is here symbolically indicated as a term with a bullet showing the position of this.

$$
\begin{aligned}
& \text { inh lookup } \quad:: \text { id } \times \text { node } \rightarrow \text { node } \\
& \text { lookup }(y, \lambda x \cdot \bullet)= \begin{cases}\operatorname{parent} & \text { if } y \equiv x \\
\operatorname{lookup}(y, \text { parent }) & \text { otherwise }\end{cases} \\
& \text { lookup }\left(y, \bullet t_{2}\right)=\operatorname{lookup}(y, \text { parent }) \\
& \text { lookup }\left(y, t_{1} \bullet\right)=\operatorname{lookup}(y, \text { parent })
\end{aligned}
$$

Fig. 1. Informal specification of name lookup

The closure analysis itself is specified as a synthesised attribute closures, which computes an overapproximation of the closures a term could evaluate to, and a collection attribute args, which computes an overapproximation of the closures a closure could be applied to. These two attributes are mutually defined as shown in Fig. 2

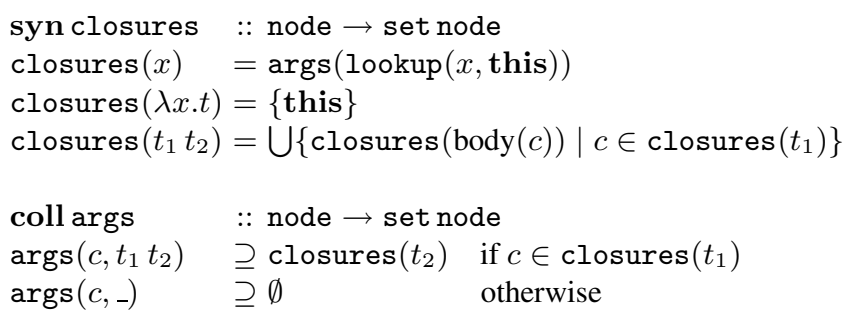

Fig. 2. Informal specification of closure analysis

The attribute closures is defined by case analysis over the content of its argument node, not its position as with attribute lookup above: this is the crucial difference between synthesised and inherited attributes. The equations are quite straightforward:

- a name can be bound to any value its binding abstraction is applied to

- a lambda abstraction's only possible value is itself

- an application's possible values are those of the body of its first child 
Note the use of reference attributes in the third equation: The attribute closures returns a set of node references, on which other attributes may in turn be evaluated.

The attribute args is a globally defined collection attribute: Every node in the tree contributes a value, and all these contributions are combined using set theoretic union to yield the attribute's final value. The attribute has type node $\rightarrow$ set node; for a closure $c, \operatorname{args}(c)$ is the set of all possible arguments $c$ could be applied to. The contribution clauses (written using $\supseteq$ ) have type node $\times$ node $\rightarrow$ set node; for a closure $c$ and a node $n$, they specify what the node $n$ contributes to the value of $\operatorname{args}(c)$. In particular, an application $t_{1} t_{2}$ with $c$ as a possible value of $t_{1}$ contributes all possible values of $t_{2}$.

The attributes closures and args circularly depend on each other, thus it is not immediately clear that this definition is sensible. The classical solution [5] is to take least fixed points, which can here be constructed by iteration: the definitions are monotonic (as we will prove) and have values over a complete partial order of finite height.

Since the inception of attribute grammars, much research has been done on how to statically detect circularities. It turns out, however, that even the very simple extension of allowing attributes to take additional parameters (like lookup) defeats all checks:

Theorem 1 (Undecidability of Circularity). It is undecidable whether a parameterised attribute is circularly defined.

Proof. See the technical report for details [25].

\section{Formalisation of Reference Attribute Grammars}

We now show how to implement reference attribute grammars, such as the example presented in the previous section, in Coq. We choose to use a shallow embedding of reference attribute grammars into Coq: Abstract syntax trees are represented as terms, with attributes as functions on them. This means that attribute equations can use the full power of the Coq language, in particular they can exploit its powerful type system.

\subsection{Formalising Abstract Syntax}

For the formalisation of the object language's abstract syntax, we make use of Ott [27], a tool for specifying the syntax and semantics of programming languages and calculi. Given an abstract grammar, Ott generates one Coq datatype per nonterminal, with one constructor per production. In our case this yields

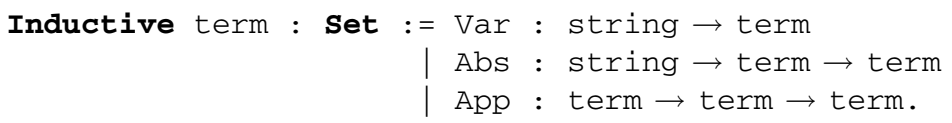

This representation, however, is not sufficient to model reference attributes. The attribute lookup, for example, should return a reference to a lambda abstraction, which is not only characterised by its content (given by term of type term), but also its position in the syntax tree to distinguish it from other abstractions that might happen to have the same body. This leads to our first implementation challenge: 
Challenge 1 (Node Identity) We need a datatype representing nodes with an identity such that they can be distinguished from other nodes, even if they have identical content.

To achieve this, we use Huet's zipper [12]. The basic idea of the zipper is to describe a node's position as a "path" from the node to the root of the tree. Let $n$ be the node under consideration. If it is the root node, its path is empty. Otherwise, $n$ must be the $i$ th child of some node $p$ and has left siblings $n_{1}, \ldots, n_{i-1}$ and right siblings $n_{i+1}, \ldots, n_{m}$, where $m$ is the total number of children of $p$. Then the path of $n$ consists of a first "step" containing all the siblings $n_{1}, \ldots, n_{i-1}, n_{i+1}, n_{m}$ (represented by their subtrees), and a path for $p$.

The possible steps are prescribed by the grammar: since every abstract syntax tree must be derivable from the start symbol, a step can only go from the occurrence of a nonterminal $t^{\prime}$ on the right hand side of some production $p$ to the nonterminal $t$ on its left hand side. In our grammar, there are only three possible steps and only one nonterminal, hence the definition of the step datatype is very simple:

$\begin{aligned} \text { Inductive step : set }: & \text { Term InApp : term } \rightarrow \text { step } \\ & \mid \text { Term } 2 \text { InApp }: \text { term } \rightarrow \text { step } \\ & \mid \text { TermInAbs : string } \rightarrow \text { step. }\end{aligned}$

The three constructors correspond exactly to the position patterns used in our informal specification of the lookup attribute in the previous section: A step Term1 InApp t2 describes (the position of) a node which is the first child of a node representing an application, and whose right sibling is $t 2-$ in the pseudocode, this was written as $\left(\bullet t_{2}\right)$. Likewise, Term2 InApp tt 1 corresponds to $\left(t_{1} \bullet\right)$ and TermInAbs $\mathrm{x}$ is $(\lambda x \cdot \bullet)$.

In order to fully describe a node's position, we need to give the complete sequence of steps all the way to the root of the syntax tree. Thus the datatype of positions is

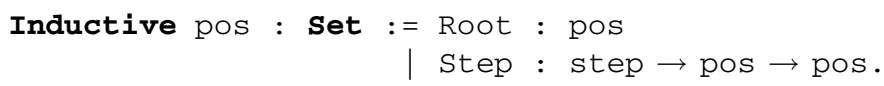

Finally, nodes are nothing but pairs of positions and content (i.e., terms), with convenience functions node_pos and node_content to access these two parts.

The example term from Sec. 2] is represented in Coq as

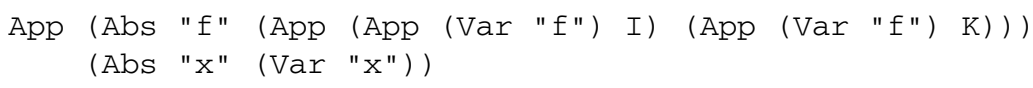

where $I$ and $\mathrm{K}$ are Coq representations of the terms $I$ and $K$, respectively. The position of the only occurrence of $x$ is

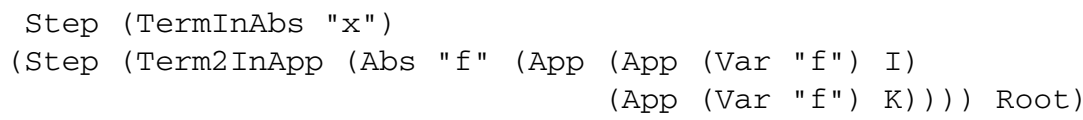

If we "plug" the content of the node into the first step on its path, we obtain the tree representation of the parent node's content. If we continue plugging all the way up to the root node, we get back the representation of the whole tree. These operations can be implemented systematically for every nonterminal as two functions plug_step and root which can be understood as tree navigation primitives for going "upwards". 
The definitions of the zipper datatypes step, pos, node, and the helper functions plug_step and root can clearly be automatically derived from the abstract grammar. Indeed, we have extended Ott to provide this facility, but since the general case of grammars with multiple nonterminals requires somewhat more involved definitions than the ones given above, we defer discussion of this feature until Sec. 4.

\subsection{Formalising Attributes}

A pleasant side effect of using a zipper-based representation of nodes is that it yields a nice characterisation of the difference between synthesised and inherited attributes: A synthesised attribute works on the first component of a node (its children), whereas an inherited attribute works on its second component (its siblings and ancestors).

We start out by rephrasing our informal specification of lookup from Sec.2 in Coq. First, we need to remedy a loophole of that specification: if we try to lookup an unbound variable, the inherited attribute described in Fig. 1 will reach the root of the syntax tree without having found any binding abstraction, and the specification does not say what is supposed to happen in this case. Our implementation of the attribute as a Coq function will have an option type as its return type, with a designated value None indicating that no binder was found.

If a binder was found, what should the function return? In the specification, it returns the binder's node. However, given our current node implementation, all nodes have type node, so we cannot by its type distinguish a node representing an abstraction from a node representing, say, an application. Hence we introduce a type that contains the same information as a node containing an abstraction, but in more explicit form 1 :

Definition closure := pos * string * term.

The type of the lookup attribute is now string $\rightarrow$ node $\rightarrow$ optionclosure. When evaluated to lookup an unbound variable, it will return None, otherwise Some $c$ where $\mathrm{c}$ is the closure representing the binding abstraction:

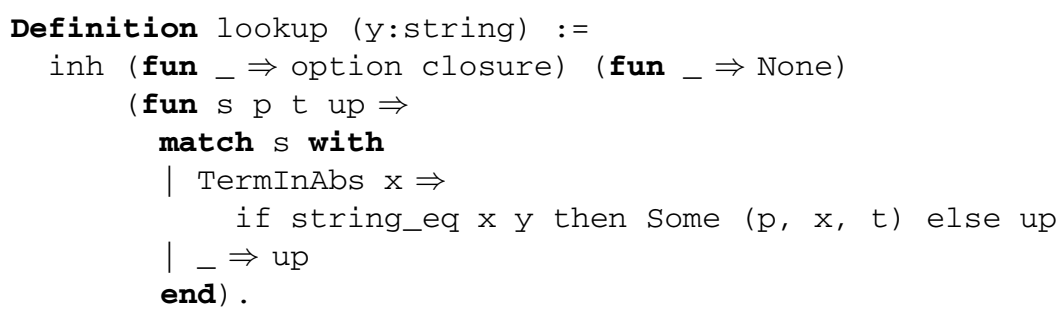

The implementation makes use of the inh combinator which takes three arguments:

1. The result type of the attribute. .

2. The default value of the inherited attribute at the root node.

3. A function to compute the attribute value on a non-root node. The four parameters of this function are

\footnotetext{
${ }^{1}$ A perhaps more elegant alternative would be to have a grammar with a separate nonterminal for abstractions; our syntax tree would then be more strongly typed, and we could directly return a node from lookup. This is the path followed in Sec.4
} 
(a) the first step of the node's position

(b) the parent node's position

(c) the contents of the node

(d) the attribute value at the parent node.

The definition of the combinator is automatically derived from the specification of the grammar by our framework. Its use greatly improves the legibility of inherited attributes; the implementation of lookup closely resembles the informal specification.

Next, we implement the synthesised attribute closures. According to its specification, it should return a set of nodes representing closures; instead of sets, our implementation uses lists (without duplicate elements). The implementation of the attribute is shown in Fig. 3 where the underlined code should be ignored for the moment. It makes use of some user-defined syntactic sugar for handling sets to improve legibility.

The collection attribute args from Sec. 2 is implemented in two parts: One is the contribution function args, which, for every node in the tree, specifies what it contributes to the value of the attribute. Its implementation is given in Fig. 3, again with some additional underlined code to be discussed shortly. The attribute closures uses the combinator coll to perform a traversal of the syntax tree, collecting contributions from all the nodes, and combining them by set union, with [ ] (the empty set) as neutral element. The combinator's definition is automatically derived from the grammar.

\subsection{Circularity}

The functions closures and args are defined by mutual recursion. However, Coq does not allow general recursion, requiring recursive functions to use well-founded recursion instead to ensure termination, which leads to our second main formalisation challenge:

Challenge 2 (Circularity) Many attributes are most easily given (mutually) circular definitions. We need a way to formalise such definitions in Coq.

In line with classical work on circular attribute grammars [58], we require all possibly circular attributes to be monotonic functions whose range is partially ordered; the actual attribute value is then computed as the least fixed point.

In our example, the attributes take values in the poset of (finite) lists of closures2. The iterative fixed point computation is formalised by giving all circularly defined attributes an extra "time to live" (TTL) parameter $t t 1$, which is a natural number that is decreased on every unsafe recursive invocation. If it ever reaches 0 , the recursion stops and [ ] (the bottom element of the poset) is returned. To achieve this, we need to introduce extra code (underlined in Fig. 3), which makes use of a convenience function to effect the "cut-off" once the counter drops to zero:

Definition TTLcut $f$ tt $1:=$ match $t t l$ with $0 \Rightarrow[]$ $S t t l^{\prime} \Rightarrow f t t l^{\prime}$ end.

This solution is quite lightweight and requires pleasantly little extra machinery.

\footnotetext{
${ }^{2}$ This partial order is a priori infinite (and of infinite height), but for any given term t the attribute closures will only return closures from $t$, which form a finite set.
} 


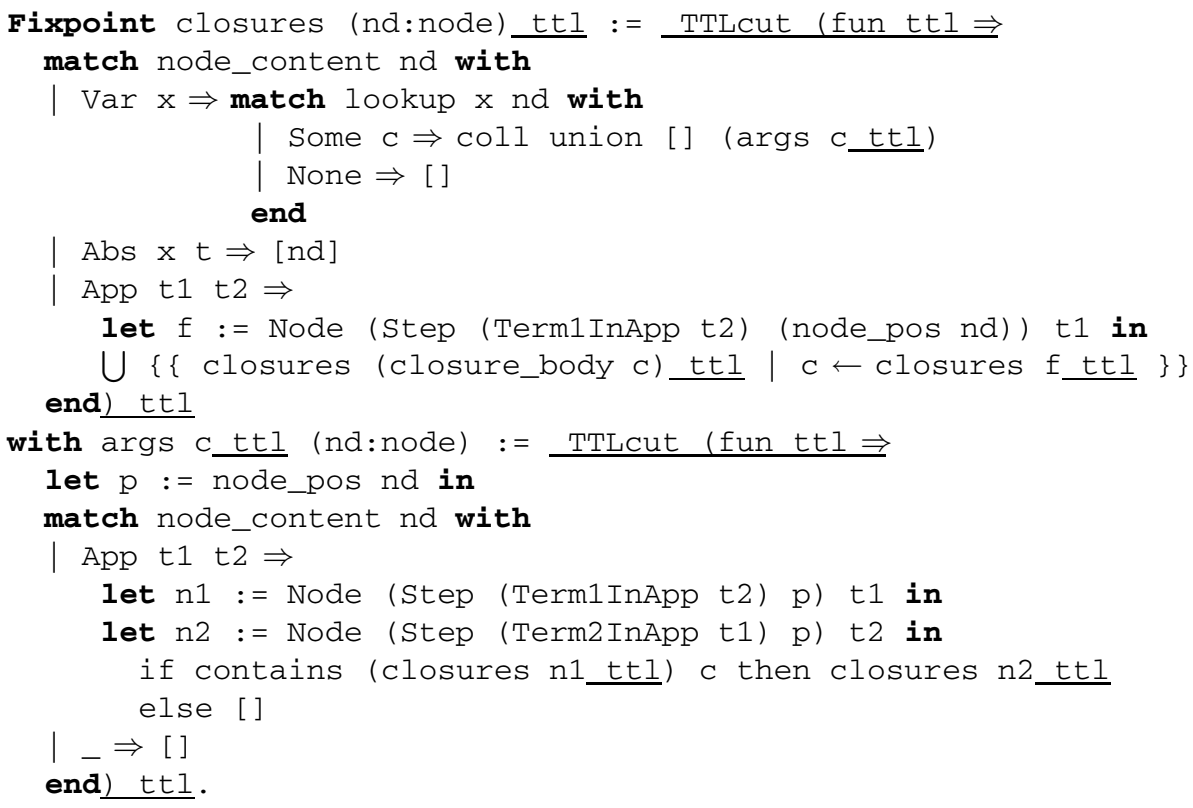

Fig. 3. Definition of closures and args; circularity handling code underlined

Our first and most urgent task is to prove monotonicity of the definitions to ensure that we can sensibly speak about their least fixed points. Since the two attributes are mutually defined, the Coq proof simultaneously establishes their monotonicity by induction on $t t 1$.

We can now proceed to the formalisation of the coherence lemma. The reduction relation on terms can be abstractly specified in Ott and is extracted to Coq as a binary predicate reduce. Lemma 1 then reads like this:

Lemma closures_sound : $\forall t \mathrm{v}$, reduce $t \mathrm{v} \rightarrow$

$\exists$ ttl, In $v$ (closures (Node Root t) ttl).

In words, this asserts that for any term $t$ and any value $v$ such that $t \Downarrow v$ there is a sufficiently large TTL value such that the analysis predicts a closure $c$ containing $v$. The proof proceeds by induction on the reduction of $t$ to $v$.

To summarise, we have shown how the implementation of closure analysis as a CRAG from Sec. 2 can be translated to Coq. The two features of reference attributes and circular attributes pose the two challenges of how to represent nodes with identity and how to accommodate non-wellfounded mutual recursion. These challenges are overcome via automatically generated datatype definitions and combinators that make it easy to translate the informal attribute specifications into Coq code, on which we can then prove the desired coherence property. 


\section{Case Study: Name Analysis}

The example in the previous section is quite simple and could easily have been written without using CRAGs. As a case study in using our framework to formalise a more substantial attribute grammar, we will now briefly discuss the formalisation of name analysis for a subset of Java. Although this is a much more complicated object language than the untyped lambda calculus, the same techniques are applicable; in fact, name analysis can be implemented using only inherited and synthesised attributes, there is no need for collection attributes. Tool support for automatic generation of definitions for the abstract syntax and the zipper datatypes is much more important now, as are carefully chosen tactics to simplify proofs. These two facilities ensure easy extensibility of the language: adding a non-naming related feature to the language usually can be done by changing only the high-level specification.

\subsection{The Grammar}

We define NameJava, a subset of Java that concentrates on the language's naming features for fields and classes, to the exclusion of almost all "operational" aspects.In particular it supports compilation units and packages, nested classes, initialisers with nested blocks, member fields and local variables, qualified names for variables and classes, and the keywords this and super.

The name lookup rules for NameJava are the same as for Java [9]; an example program that highlights some of the salient points appears in Fig. 4. The five local variables a1 to a 5 are assigned as follows: $a 1$ is bound to the local variable a, a 2 to the field a of class D, a 3 to the field a of class B, a 4 to the field a of class B (since the local variable $A$ obscures the class of the same name), and a 5 to the field a of class $A$.

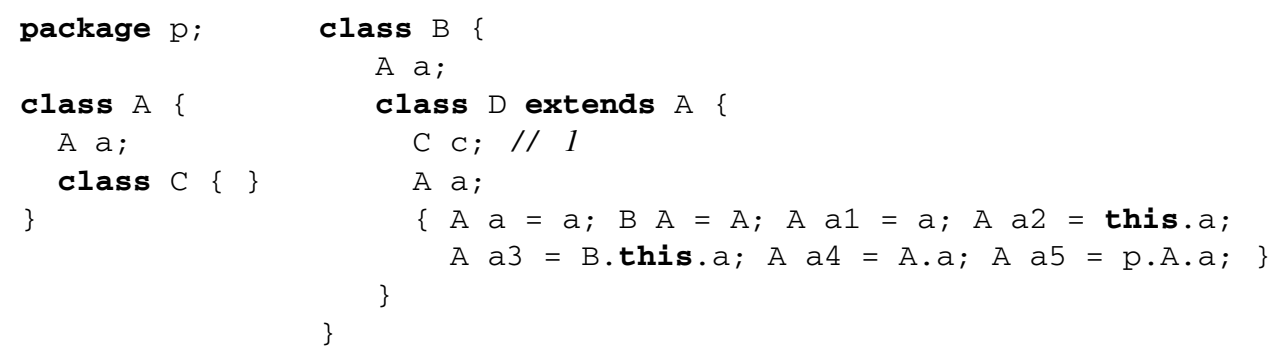

Fig. 4. Example NameJava program

The grammar of NameJava is, as for the untyped lambda calculus, specified in Ott, so datatype definitions for the abstract syntax and the zipper are automatically generated. For example, the inductive datatype for programs is

Inductive program : Set := CProgram : list compunit $\rightarrow$ program. expressing that a program is a list of compilation units. 
For the zipper datatypes, our automatic extraction actually generates a more strongly typed version than the one shown in Section 2, there is not a single datatype node of nodes, but rather a family of datatypes indexed by the nonterminal they represent. The index type is an enumeration type with one constructor per nonterminal of the grammar:

Inductive nt : Set := Program | Compunit | Classdecl | Extclause

| Bodydecl | Block | Stmt | Vardecl | Expr | Access | ..

The datatype of steps is doubly indexed over nt. A step $t^{\prime} t$ describes the position of a subtree derived from nonterminal $t$ ' within its parent, which is derived from nonterminal $t$, i.e. it is a "step from $t$ ' to $t$ ". We cannot give the definition of step for NameJava in full due to space constraints, but a fairly typical constructor is

BodydeclInclassdecl : string $\rightarrow$ extclause $\rightarrow$
$\quad$ list bodydecl $\rightarrow$ list bodydecl $\rightarrow$ step Bodydecl Classdecl

describing the position of a body declaration within a class declaration: To reconstruct the complete class declaration given one of its body declarations $b$, we need to know the class name (a string), the extends clause, the body declarations to the left of $b$, and the body declarations to the right of $b$ - exactly the data stored in the step constructor.

As before, the pos datatype is a sequence of steps. It is, however, likewise indexed over a nonterminal (thus pos $t$ describes the type of positions of subtrees derived from $t$ ), and it ensures that the steps it is composed of "fit together":

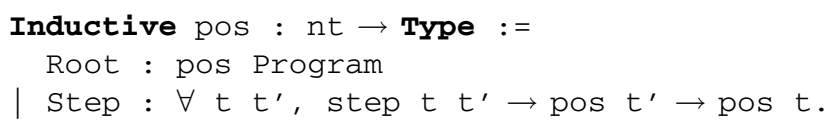

Finally, to define the type of nodes, we need to ensure that the content of a node $t$ actually is of the right type, i.e., a term of the inductive datatype corresponding to the index $t$. To this end we make use of an (automatically generated) function content, that maps indices to datatypes - for example, content Program is program:

\footnotetext{
Inductive node : nodetype $\rightarrow$ Type :=

Node $: \forall t$, pos $t \rightarrow$ content $t \rightarrow$ node $t$.
}

The upshot of using this more complicated, indexed representation of zippers is that it rules out many positions and nodes that could never occur in a well-formed program. For example, with the given NameJava grammar we can prove (in Coq) that the parent of a node Stmt will always be a node Block. This is crucial for the definition of plug_step and root, which both map nodes to trees; if the nodes were improperly nested, no corresponding trees could be constructed.

\subsection{Attributes for Name Analysis}

Name lookup in NameJava proceeds basically in an "outwards" movement, searching through enclosing lexical scopes until a matching declaration is found. Member classes and fields, however, can also be inherited from an ancestor class, hence an "upwards" movement (up the inheritance chain) is superimposed on the underlying outwards movement. Qualified names, finally, contribute a third type of movement: first, the declaration of the qualifier (resp. its type if it is a variable) is looked up, then the 
qualified name is looked up as a member within this declaration (again possibly following the inheritance chain upwards, but never going outwards through enclosing scopes).

Name lookup for classes is implemented using five attributes, modelled after the implementation in the JastAddJ Java compiler [7]:

- toplevel_class looks up a toplevel class within a compilation unit

- canonical_class looks up a class by name and package

- local_class looks up a local member class within a class

- member_class looks up a member class in a class or one of its ancestors

- lookup_class looks up a class, starting from an arbitrary node and searching through enclosing scopes

The first three of these just iterate over a list of declarations, looking for the first one with the right name. The behaviour of lookup_class and member_class and their interaction, is more interesting, since they circularly depend on each other: Suppose that lookup_class is used to lookup the type name " $\mathrm{C}$ " from the line marked with " 1 " in Fig. 4 First, class D is searched for a member_class named C. Member classes include both locally defined classes and inherited member classes. Since no local_class C exists, we need to invoke member_class on D's super class.

To resolve the super class, we need to lookup the name "A " by lookup_class, causing member_class to depend on lookup_class. That lookup will search for a member_class named " $A$ " in the enclosing class $B$, causing lookup_class to depend on member_class in turn. Since $A$ is not a member class of B, it will be found as a toplevel_class. Once we have a reference to A, class $C$ is successfully looked up as member_class of A.

Observe that this last step makes crucial use of reference attributes: The node of class $A$ is retrieved as the value of attribute lookup_class, and then attribute member_class is in turn evaluated on it. The example also shows that the two attributes recursively invoke each other, and thus are circularly defined.

\subsection{Circularity in Name Analysis}

Nevertheless, this circularity is quite different from the one encountered when implementing closure analysis: Whereas the specification of closure analysis is genuinely circular and the actual result of the analysis is the least fixed point of the given equations, the circularity of the definitions of lookup_class and member_class is somewhat accidental. We would, in fact, hope that the mutual recursive invocations of these two attributes are well-founded so that the attribute evaluation eventually terminates.

However, the language described so far allows classes that extend themselves, i.e. definitions like class $A$ extends $A\{\}$. Looking up a member in $A$ will lead the two attributes to repeatedly invoking each other on the same arguments, and thus to nontermination. The Java Language Specification [9] forbids programs like the above: Well-formed Java programs must not contain classes that are their own descendants.

It is clear that lookup on classes with acyclic inheritance hierarchy always terminates: When member_class recursively invokes itself on the superclass (as determined by lookup_class), we have moved up one level of inheritance, so eventually we will arrive at a class that extends no other class, and the member lookup will terminate. So 
in order to implement a version of lookup_class that is guaranteed to terminate, we need to require that the node it is invoked on belongs to a program with acyclic inheritance hierarchy. However, in order to define the concept of an acyclic inheritance hierarchy we need a definition of inheritance, and for this we need lookup_class!

There does not seem to be an easy solution to this chicken-and-egg problem, so we accept the presence of a possible circularity, and treat it in the same way as for closure analysis: The circularly defined attributes take an additional TTL parameter and produce values over the flat poset of optional values, defined by the Coq type option, with None standing for nontermination.

Once we have implemented class lookup as a partial function, we can define what it means for a class to have an acyclic inheritance hierarchy, and we can give a (constructive) proof that when looking up a member class of such a class, there is a sufficiently large number $n$ for which lookup will always return a value when invoked with $n$ as its TTL counter. In other words, we can now prove that member lookup on classes with acyclic inheritance hierarchy always terminates.

Note that the need for circularly defined class lookup arises from the presence of nested classes. In a language without nested classes, all classes are toplevel classes within compilation units, so class lookup could be implemented by the two attributes toplevel_class and canonical_class alone, without any circularity.

\subsection{Towards the Verification of Rename Refactorings}

We cannot prove the correctness of our lookup implementation, since there is no formal specification to validate it against, so we apply our framework to a different problem: In [24], we describe how the well-known Rename refactoring, i.e. changing the name of a declaration and all its uses, can be implemented using right inverses to the lookup functions. We start from the observation that a reasonable correctness criterion for a renaming would be that the "binding structure" of the program does not change, i.e. all names in the program still refer to the same declarations as before.

We therefore define an access computation function that is a right inverse to lookup: Whereas lookup computes the declaration $d$ a name $n$ binds to at a program location $p$, access computes a name $n$ that a declaration $d$ can be accessed under from a location $p$, where $n$ might be qualified to avoid capture. The inversion property means that if access gives a name $n$ to access $d$ from $p$, looking up $n$ from $p$ will yield $d$.

Given an implementation of access computation with this property, it is, in principle, easy to implement a rename refactoring: Given an input program, we first determine which declaration every individual name refers to. Then we perform the requested name change. Now we go over the program again. For every name, we know the declaration it ought to refer to, and if it does not do so anymore we can use access computation to determine an alternative name that does, and replace the old name with it.

In our current case study we concentrate on proving the inversion property. Given the implementation of the attributes for class and variable lookup, we can write corresponding access computation attributes. For member_class, which looks up a member class, we have an attribute access_member, which constructs an access to a member class; for lookup_class we have access_class, and so on. The inversion 
of an attribute definition is quite systematic, and it is easily verified that all the access computation attributes are indeed right inverse to their corresponding lookup attributes.

Many of the proofs proceed by induction on either trees or positions, and hence have to handle (at least) one case per language construct to establish the induction step. Only a limited number of cases is actually interesting for every given proof, however: When proving properties of name lookup, for example, only constructs corresponding to naming scopes need special attention, in all the other cases the induction hypothesis can be applied directly. This situation can be handled gracefully by the Coq tactic language: When doing an induction, we specify a default tactic to be tried on all new subgoals; usually, Coq's auto tactic is sufficient to discharge all the trivial cases, if its hint database has been populated with enough domain specific lemmas.

\section{Evaluation}

\subsection{Statistics}

Figure 5 gives some statistics about the size of the complete Coq development for both our examples: the implementation of closure analysis in Sec. 3 and the name analysis for NameJava in Sec. 4, For both we give the line count for the Ott specification and for the Coq code automatically extracted from that specification, written as a sum: The first summand represents the size of the code extracted by Ott, and the second the size of the code extracted by our extension. Furthermore, we give the line count for the handwritten Coq specification, and for the proof scripts, as well as the total line count.

\begin{tabular}{l||r|r} 
& Closure Analysis & NameJava \\
\hline Ott specification (LOC) & 80 & 322 \\
extracted Coq code (LOC) & $118+163$ & $403+443$ \\
Coq specification (LOC) & 301 & 2219 \\
proof scripts (LOC) & 233 & 1694 \\
\hline total LOC & $\mathbf{6 1 4}$ & $\mathbf{4 2 3 5}$
\end{tabular}

Fig. 5. Size of development

As expected, the name analysis is far bigger than the closure analysis, at almost seven times the size. In both cases, we have profited clearly from writing a high-level specification in Ott and automatically extracting the definitions of tree and zipper datatypes: the Ott specification is only about a third of the size of the extracted code. It is worth noting, however, that we use Ott merely as a convenient language for describing the object language syntax. All the proofs are carried out inside Coq, on the generated abstract syntax, and are fully certified by the Coq kernel.

It is interesting to see that the proof scripts are smaller than the specification they verify, only comprising about $40 \%$ of the total line count on both projects. This number is similar to other formalisation projects, such as Leroy's CompCert [19]. 


\subsection{Extensibility}

Although NameJava has all the major naming related features of full Java, it is a much smaller language. We have shown how the same implementation techniques can be used to specify closure analysis on the lambda calculus and to prove the inversion property of access computation and lookup in NameJava. But what about extending to a larger subset of Java, or even the full language? Will all the proofs have to be redone?

Fortunately, the answer is no. Adding a non-naming related feature to the language is possible without breaking any of the proofs: Introducing an if statement to the language only needs five additional lines of Ott. Neither lookup nor access computation have to be changed, and all the proofs still go through unchanged. For language features such as the for loop, which do influence the lookup without introducing any new naming related concepts, the effort is still moderate (40 lines of specification, 50 lines of proof). Adding, e.g., interfaces would certainly require more effort, but the experience of JastAddJ shows that CRAGs are powerful enough to handle the full Java language.

\section{Related Work}

Proofs of analyses and transformations. Cachera et al. [4] derive a dataflow analysis in Coq by abstract interpretation, independent of an implementation language. On the other end of the spectrum, Lerner et al. [18] introduce a domain specific language to encode analyses, which are then automatically proved correct. Our work is positioned in the middle: Using CRAGs offers a convenient implementation language, which is nevertheless fully integrated with Coq; the price we pay is less automation.

Formalising attribute grammars. Traditionally, attribute grammars have been encoded in lazy functional languages as families of functions, one per nonterminal, that take inherited attributes as parameters and return synthesised attributes as results [15]. Our method represents different attributes by different functions, thus staying closer to the original attribute grammar. It is not clear if the traditional encoding could accommodate reference attributes. The zipper has been used before to implement inherited attributes [29]2], but without making the connection to reference attributes.

Formalisations of Java. There has been much interest in the formalisation of (subsets of) the Java language [14|21], but most formalisations focus on the semantics of the language and mostly ignore more syntactic aspects like lookup rules. Only FJI [13] makes an effort to model nested classes, but the formalisation is geared towards minimality and does not give a satisfying solution to the name lookup circularity problem.

\section{Conclusions}

We have presented a novel framework for formalisation and verification of analyses and transformations that are expressed at source level, rather than on a simplified intermediate language. The key to this framework are circular reference attribute grammars, which have proven their practical worth in compiler generation tools [1 231877].

We have presented a general method for formalising them in Coq, enabling the expression of specifications and proofs at a high level of abstraction. We provide tools 
that automatically generate datatype definitions and utility functions from a high-level description of the object language, which are complemented by domain-specific tactics to simplify proofs. We have validated these tools and tactics with a non-trivial case study, namely renaming in the presence of the complex binding rules of modern objectoriented languages. The verification effort is manageable, and the proofs are scalable, so that they do not have to be redone from scratch when the object language changes.

As future work, we would like to explore the implementation of a domain specific language for attribute grammars on top of Coq. This would give additional possibilities for automation, and it might be possible to support automatic proofs of common lemmas such as the monotonicity of circularly defined attributes. Still, we feel that it is essential to provide close integration of the attribute implementations with the Coq language in general, so as not to lock the user into the DSL, since the more complex proofs can likely not be fully automated and certainly benefit from the power of the full system.

We hope this paper has enthused others to venture into the formalisation and verification of source level analyses. Modern development tools need to stay at the source level to interact with the user, but bugs are common even in production quality environments. Our work shows that rigorous verification of the underlying analyses is feasible, which can help in improving tools used by developers on a daily basis.

\section{References}

1. Augusteijn, L.: Definition of the programming language Elegant, 2000. Release 7.1, Philips Research Laboratories, Eindhoven, the Netherlands (February 2000)

2. Badouel, É., Fotsing, B., Tchougong, R.: Yet Another Implementation of Attribute Evaluation. Technical Report 6315, INRIA Rennes (2007)

3. Boyland, J.T.: Remote attribute grammars. J. ACM 52(4), 627-687 (2005)

4. Cachera, D., Jensen, T., Pichardie, D., Rusu, V.: Extracting a data flow analyser in constructive logic. Theor. Comput. Sci. 342(1), 56-78 (2005)

5. Chirica, L.M., Martin, D.F.: An Order-Algebraic Definition of Knuthian Semantics. Mathematical Systems Theory 13, 1-27 (1979)

6. Ekman, T., Ettinger, R., Schäfer, M., Verbaere, M.: Refactoring bugs (2008), http:// progtools.comlab.ox.ac.uk/refactoring/bugreports

7. Ekman, T., Hedin, G.: The JastAdd Extensible Java Compiler. In: OOPSLA (2007)

8. Farrow, R.: Automatic generation of fixed-point-finding evaluators for circular, but welldefined, attribute grammars. In: CC, pp. 85-98 (1986)

9. Gosling, J., Joy, B., Steele, G., Bracha, G.: The Java Language Specification (2005)

10. Hanson, D., Proebsting, T.: A research C\# compiler. SPE 34(13) (2004)

11. Hedin, G.: Reference Attributed Grammars. Informatica (24), 301-317 (2000)

12. Huet, G.P.: The Zipper. J. Funct. Program. 7(5), 549-554 (1997)

13. Igarashi, A., Pierce, B.C.: On inner classes. Inf. and Comp. 177(1), 56-89 (2002)

14. Igarashi, A., Pierce, B.C., Wadler, P.: Featherweight Java: A Minimal Core Calculus for Java and GJ. In: OOPSLA, pp. 132-146 (1999)

15. Johnsson, T.: Attribute grammars as a functional programming paradigm. In: Kahn, G. (ed.) FPCA 1987. LNCS, vol. 274, pp. 154-173. Springer, Heidelberg (1987)

16. Knuth, D.E.: Semantics of context-free languages. Mathematical Systems Theory 2, 127-146 (1968); correction: Mathematical Systems Theory 5, 95-96 (1971)

17. Lacey, D., Jones, N.D., Van Wyk, E., Frederiksen, C.C.: Proving Correctness of Compiler Optimizations by Temporal Logic. In: POPL, pp. 283-294. ACM Press, New York (2002) 
18. Lerner, S., Millstein, T., Rice, E., Chambers, C.: Automated soundness proofs for dataflow analyses and transformations via local rules. SIGPLAN Not. 40(1), 364-377 (2005)

19. Leroy, X.: Formal certification of a compiler back-end. In: POPL, pp. $42-54$ (2006)

20. Magnusson, E., Hedin, G.: Circular Reference Attributed Grammars. Sci. Comput. Program. 68(1), 21-37 (2007)

21. von Oheimb, D., Nipkow, T.: Machine-checking the java specification: Proving type-safety. In: Alves-Foss, J. (ed.) Formal Syntax and Semantics of Java. LNCS, vol. 1523, p. 119. Springer, Heidelberg (1999)

22. Palsberg, J.: Closure Analysis in Constraint Form. TOPLAS 17(1), 47-62 (1995)

23. Reps, T.W., Teitelbaum, T.: The Synthesizer Generator: A system for constructing languagebased editors. In: Texts and Monographs in Computer Science. Springer, Heidelberg (1989)

24. Schäfer, M., Ekman, T., de Moor, O.: Sound and Extensible Renaming for Java. In: Kiczales, G. (ed.) OOPSLA. ACM Press, New York (2008)

25. Schäfer, M., Ekman, T., de Moor, O.: Formalising and Verifying Reference Attribute Grammars in Coq (Technical Report and Implementation) (2009), http: / progtools . comlab.ox.ac.uk/projects/refactoring/formalising-rags

26. Sestoft, P.: Analysis and Efficient Implementation of Funct. Prog. Ph.D. thesis (1991)

27. Sewell, P., Nardelli, F.Z., Owens, S., Peskine, G., Ridge, T., Sarkar, S., Strnisa, R.: Ott: effective tool support for the working semanticist. In: ICFP, pp. 1-12 (2007)

28. Ekman, T.: JastAdd (2008), http: / / www . jastadd. org

29. Uustalu, T., Vene, V.: Comonadic functional attribute evaluation. In: TFP (2005) 